Network Working Group

Request for Comments: 3933

J. Klensin

BCP : 93

Category: Best Current Practice

S. Dawkins

November 2004

\title{
A Model for IETF Process Experiments
}

Status of this Memo

This document specifies an Internet Best Current Practices for the Internet Community, and requests discussion and suggestions for improvements. Distribution of this memo is unlimited.

Copyright Notice

Copyright (C) The Internet Society (2004).

Abstract

The IETF has designed process changes over the last ten years in one of two ways: announcement by the IESG, sometimes based on informal agreements with limited community involvement and awareness, and formal use of the same mechanism used for protocol specification. The first mechanism has often proven to be too lightweight, the second too heavyweight.

This document specifies a middle-ground approach to the system of making changes to IETF process, one that relies heavily on a "propose and carry out an experiment, evaluate the experiment, and then establish permanent procedures based on operational experience" model rather than those previously attempted.

Table of Contents

1. Introduction . . . . . . . . . . . . . . . . . . . . . 2

2. Background and Specification . . . . . . . . . . . . . . 2

3. Security Considerations . . . . . . . . . . . . . . . . . . . 5

4. Acknowledgements . . . . . . . . . . . . . . . . . . 5

5. References . . . . . . . . . . . . . . . . . . . . . 5 5.1. Normative Reference. . . . . . . . . . . . . . . . 5 5.2. Informative References. . . . . . . . . . . . . 5

6. Authors' Addresses . . . . . . . . . . . . . . . . 6 Full Copyright Statement . . . . . . . . . . . . . . . 7 


\section{Introduction}

This document specifies a middle-ground approach to the system of making changes to IETF process, one that relies heavily on a "propose and carry out an experiment, evaluate the experiment, and then establish permanent procedures based on operational experience" model rather than those previously attempted.

\section{Background and Specification}

Since the 1992 changes documented in [RFC1396], the IETF has used two mechanisms for process changes.

1. IESG has adopted a number of procedural changes on its own initiative and documented them informally, utilizing the wide discretion implicitly granted to them by [RFC2026]. This provided a lightweight mechanism for change, but the lightness came with a cost: There was sometimes too little alignment with the larger IETF community.

2. The IETF has also used the [RFC2026] protocol standards development process to identify a community of interest, hold one or more BoFs, charter a working group, discuss proposed changes within the community, develop IETF-wide consensus on the changes, and publish (usually) Best Current Practice specifications. This provided full community involvement but also came with a cost in flexibility. The IETF does not change its formal processes often (the IPR clarifications in [RFC3667, RFC3668] are the first documented changes to [RFC2026] since 1996), and the community is understandably reluctant to permanently alter or extend formally adopted processes with untried new procedures.

There is a middle ground between BCP process updates and informal agreements. This document specifies regularizing and formalizing the informal IESG mechanisms listed in 1 above as a means of moving forward with procedural changes that might prove valuable.

The mechanisms outlined here add to the IESG's range of tools for dealing with process issues on an ongoing basis. They supplement the existing tools rather than attempting to replace them with a single "magic bullet". The choice of using the procedure outlined in this document or other mechanisms available to the IESG and the community -- present or future -- remains in the IESG's hands. If the IESG does not exercise this discretion wisely, this document provides no additional remedies. 
Some have interpreted the current procedures as giving the IESG all of the capabilities outlined here. If this were true, this document only encourages the IESG to use this type of mechanism more frequently in preference to less streamlined ones, and to more explicitly document its actions and decisions.

This specification permits and encourages the IESG to adopt and institute "process experiments" by using the following procedure:

1. An I-D is written describing the proposed new or altered procedure. A statement of the problem expected to be resolved is desirable but not required (the intent is to keep the firm requirements for such an experiment as lightweight as possible). Similarly, specific experimental or evaluative criteria, although highly desirable, are not required -- for some of the process changes we anticipate, having the IESG reach a conclusion at the end of the sunset period that the community generally believes things to be better (or worse) will be both adequate and sufficient. The I-D must state an explicit "sunset" timeout typically, not to exceed one year after adoption.

2. If the IESG believes the proposal is plausible and plausibly useful, a four-week IETF last Call is initiated. The IESG can institute whatever procedures it wishes to make this determination and to avoid denial of service attacks from large numbers of spurious or unimportant proposals. In particular, they might institute a procedure requiring a number of endorsements, or endorsements of a particular type, before the IESG considers the proposal. The IESG is, however, expected to understand that procedures or review processes that act as a mechanism for significant delays do not fall within the intent of this specification.

3. At the conclusion of the Last Call, the IESG reevaluates the plausibility and appropriateness of the proposal. If they conclude that the proposed experiment is appropriate, a second I-D is generated (either by the IESG or by the original authors with IESG advice) that cleans up any definitional issues exposed in the Last Call and that explicitly identifies and responds to issues raised during the Last Call.

4. The document and experiment are then announced, the experiment is begun, and the document is forwarded for publication as an Experimental RFC. 
The IESG is explicitly authorized to use this mechanism (based on Experimental RFCs) to gain experience with proposed changes to $B C P$ specifications. There is no requirement to approve a $\mathrm{BCP}$ specification for the experiment until the experiment is found to have value.

The IESG could, of course, reach a "bad idea" conclusion at any stage in this process and abandon the experiment. It might recommend publication of the experimental document, with a discussion of why it was a bad idea, but is not required to do so. The list above is deliberately vague about where the I-Ds come from: a WG, design team, individual contribution, editing group, or other mechanism could be used in the first and/or third steps, but no specific mechanisms are required, and the IESG is explicitly permitted to generate such proposals internally.

In each case, the IESG's decision to go forward (or not) with a procedural experiment, or the steps leading up to one, is expected to reflect their judgment of the existence of rough consensus in the community. That judgment may be appealed using the usual procedures, but the IESG and the community are reminded that an experimental attempt to try something for a fixed period is typically a better engineering approach than extended philosophical discussion without any experience to back it up.

Nothing above is to be construed as requiring an IETF-wide attempt for any given process experiment. A proposal for such an experiment may specify selected areas, selected working groups, working groups meeting some specific criteria (e.g., those created after a particular time or of a specified age), or be specific in other ways.

At or before the end of the "sunset" timeout, the IESG would either revise (or cause to be revised) the document into a BCP RFC or the procedure would expire and, presumably, not be tried again unless something changed radically. A document describing why the experiment had succeeded or failed would be desirable but could not, realistically, be a requirement. If the procedure went to $\mathrm{BCP}$, the $\mathrm{BCP}$ would reflect what we would call "operational experience" in the real world.

We note that, if the procedures the IESG has adopted (and the procedural exceptions it has made) over the last decade are legitimate, then the IESG has the authority to institute the changes specified here by bootstrapping this process. 


\section{Security Considerations}

This document specifies a mechanism for evolving IETF procedures. It does not raise or consider any protocol-specific security issues. In considering experimental changes to procedures, the IESG should, of course, exercise due caution that such changes not reduce the quality of security review and consideration for protocols or, at least, that the process experiment proposals contain early detection and correction mechanisms should quality deterioration occur.

4. Acknowledgements

The first revision of this document benefited significantly from suggestions and comments from Avri Doria, Margaret Wasserman, and Harald Alvestrand, and from discussions with the General Area Directorate and at its open meeting during IETF 59. After mailing list discussion, considerable explanatory material was removed to a separate document for the current version.

The first version of this document was posted as an Internet Draft on February 7, 2004 .

5. References

5.1. Normative References

[RFC2026] Bradner, S., "The Internet Standards Process -- Revision 3", BCP 9, RFC 2026, October 1996.

\subsection{Informative References}

[RFC1396] Crocker, S., "The Process for Organization of Internet Standards Working Group (POISED)", RFC 1396, January 1993.

[RFC3667] Bradner, S., "IETF Rights in Contributions", BCP 78, RFC 3667, February 2004 .

[RFC3668] Bradner, S., "Intellectual Property Rights in IETF Technology", BCP 79, RFC 3668, February 2004. 
6. Authors' Addresses

John C Klensin

1770 Massachusetts Ave, \#322

Cambridge, MA 02140

USA

Phone: +1 6174915735

EMail: john-ietf@jck.com

Spencer Dawkins

1547 Rivercrest Blvd.

Allen, TX 75002

USA

Phone: +1 4693303616

EMail: spencer@mcsr-labs.org 
Full Copyright statement

Copyright (C) The Internet Society (2004).

This document is subject to the rights, licenses and restrictions contained in BCP 78, and at www.rfC-editor.org, and except as set forth therein, the authors retain all their rights.

This document and the information contained herein are provided on an "AS IS" basis and THE CONTRIBUTOR, THE ORGANIZATION HE/SHE REPRESENTS OR IS SPONSORED BY (IF ANY), THE INTERNET SOCIETY AND THE INTERNET ENGINEERING TASK FORCE DISCLAIM ALL WARRANTIES, EXPRESS OR IMPLIED, INCLUDING BUT NOT LIMITED TO ANY WARRANTY THAT THE USE OF THE INFORMATION HEREIN WILL NOT INFRINGE ANY RIGHTS OR ANY IMPLIED WARRANTIES OF MERCHANTABILITY OR FITNESS FOR A PARTICULAR PURPOSE.

Intellectual Property

The IETF takes no position regarding the validity or scope of any Intellectual property Rights or other rights that might be claimed to pertain to the implementation or use of the technology described in this document or the extent to which any license under such rights might or might not be available; nor does it represent that it has made any independent effort to identify any such rights. Information on the ISOC's procedures with respect to rights in ISOC Documents can be found in BCP 78 and BCP 79 .

Copies of IPR disclosures made to the IETF Secretariat and any assurances of licenses to be made available, or the result of an attempt made to obtain a general license or permission for the use of such proprietary rights by implementers or users of this specification can be obtained from the IETF on-line IPR repository at http://www.ietf.org/ipr.

The IETF invites any interested party to bring to its attention any copyrights, patents or patent applications, or other proprietary rights that may cover technology that may be required to implement this standard. Please address the information to the IETF at ietfipreietf.org.

Acknowledgement

Funding for the RFC Editor function is currently provided by the Internet Society. 